\title{
Surface Analysis Correlated with Structural and Mechanical Properties of Laser Irradiated Brass
}

\author{
Shahbaz Ahmad*, Shazia Bashir, Daniel Yousaf, Nisar Ali, Tousif Hussain \\ Center for Advanced Studies in Physics, Government College University, Lahore, Pakistan \\ Email: ${ }^{*}$ shahbazcasp@gmail.com
}

Received 17 October 2014; revised 10 November 2014; accepted 8 December 2014

Copyright (C) 2015 by authors and Scientific Research Publishing Inc.

This work is licensed under the Creative Commons Attribution International License (CC BY). http://creativecommons.org/licenses/by/4.0/

cC) (7) Open Access

\begin{abstract}
Brass targets were irradiated with various laser pulses of Excimer laser ranging from 1200 to 3000 for constant fluence of $3.4 \mathrm{~J} / \mathrm{cm}^{2}$ in oxygen atmosphere (100 Torr). The surface morphology and crystallographic analyses were performed by using Scanning Electron Microscope (SEM) and $\mathrm{X}$-Ray Diffractometer (XRD). SEM analysis reveals the formation of laser-induced micro-sized cavities, bumps, cones and wave-like ridges with non-uniform shape and density distribution. These features are formed for all number of pulses; however with increasing number of pulses from 1200 to 2400, the density of cavities decreases whereas, the wave-like ridges become more pronounced and bump-formation is vanished. For maximum number of 3000 shots, the appearance of cones and wave-like ridges becomes diffusive, whereas the density and size of cavities increase again. XRD analysis demonstrates that no new phases are formed in irradiated brass. However, the change in peak intensity along with lower and higher angle shifting is observed which is attributed to generation of laser induced stresses. The Yield Stress (YS), Ultimate Tensile Strength (UTS) as well as Microhardness increase monotonically with increasing number of laser pulses.
\end{abstract}

\section{Keywords}

Laser Pulses, Surface Morphology, Mechanical Properties, Crystallographic Alteration

\section{Introduction}

The surface, structural and mechanical properties (yield stress, tensile strength and hardness) of metals and their alloys can be significantly improved by employing a variety of techniques such as ion beam modification, laser

*Corresponding author.

How to cite this paper: Ahmad, S., Bashir, S., Yousaf, D., Ali, N. and Hussain, T. (2015) Surface Analysis Correlated with Structural and Mechanical Properties of Laser Irradiated Brass. Materials Sciences and Applications, 6, 23-32. 
assisted ablation or deposition, plasma processing [1]-[4]. Laser surface engineering is one of an efficient and reliable technique for material processing applications. Reduced heat affected zone, control over the laser fluence, speed, versatility, non-contact nature, contamination free and environmental friendly ablation process are the advantages of laser processing method over other techniques [3].

The surface, structural and mechanical properties e.g. yield stress, tensile strength and hardness of brass can be improved by laser irradiation. The substantial quantity of work is reported on laser irradiation of metals in different environments [5]-[7]. Bashir et al. [5] have investigated the effect of laser pulses and ambient environment (dry or wet) and on the laser ablation performance of brass. Their results proposed that ambient environment plays a significant role in surface and structural modification of brass. Kazakevich et al. [7] prepared brass nanoparticles by brass target ablation in ethanol using copper-vapor laser radiation at various laser fluences. They found that the surface morphology of the irradiated brass target strongly influence the properties of laser-generated nanoparticles. Tam et al. [8] modified the brass surface using a 2 kW CW Nd-YAG laser. For modified layers the hardness was increased from $110 \mathrm{HV}$ to the range of $152-256 \mathrm{HV}$.

In the previous [9] work our group has investigated the effect of carbon ion produced by Pelletron accelerator on the same target. In this work ions of constant of $2 \mathrm{MeV}$ energy with various fluences ranging from $56 \times 10^{12}$ to $26 \times 10^{13}$ ions $/ \mathrm{cm}^{2}$ were irradiated on brass target. These ions can transfer their energy to the lattice atoms. If energy of host atom is greater than displacement threshold then the host atom displaced from normal lattice site. This displaced atom is called primary knocked atom (PKA). A sufficient amount of the energy is transferred to the PKA, as a result PKA produces displacement cascades. Therefore, large numbers of vacancy-interstitial defects (Frenkel pair or Frenkel defect) are produced. Both the incident ions and PKA give rise to a number of effects at the surface and in subsurface area. These effects and defects bring alteration in structural and mechanical properties of irradiated brass. The present work deals with laser irradiation effect on surface, structural and mechanical behavior of brass. The main processes included are multi photon ionization and inverse bremsstrahlung. These effects produce thermal and pressure gradient on the material, as a result many defects are generated in the normal lattice site of host atoms. These laser induced defects produce modifications in structural and mechanical properties of brass. These results can be compared with ion irradiation results as reported previously [9].

In the present work the effect of nanosecond laser pulses on the surface, structural and mechanical properties of brass has been investigated. The targets were exposed to Excimer laser of wavelength $248 \mathrm{~nm}$ with constant fluence of $3.4 \mathrm{~J} / \mathrm{cm}^{2}$ under ambient environment of oxygen. The surface morphology, crystallographical analysis, tensile testing and surface hardness were performed by using SEM, XRD, UTM techniques and Vicker hardness tester respectively.

\section{Experimental Setup}

KrF Excimer laser (EX GAM USA 200) with the $248 \mathrm{~nm}$ wavelength, $20 \mathrm{~ns}$ pulse duration, $150 \mathrm{~mJ}$ maximum pulse energy was employed to conduct the experiments. Rectangular shaped commercial grade brass with dimension $45 \times 6 \times 3 \mathrm{~mm}$ was used as a target. Prior to laser treatments, the specimens were grinded and polished with Silicon Carbide (SiC) papers of different progressive grades. The samples were washed during grinding with acetone to remove the impurities. The grinded specimens were sealed into Pyrex glass tube, evacuated up to a base pressure of $10^{-6}$ Torr by using rotary pump followed by diffusion pump. In order to relieve internal stresses and defects the specimens were annealed in a muffle furnace (Nabertherm-LHT-02/18, Germany) at 773 $\mathrm{K}$ for 2 hours. After annealing, the specimens were cleaned ultrasonically with acetone for 30 minute and mounted on a sample holder attached to DC motor. The mounted samples were placed in UHV chamber. The chamber was evacuated to a base pressure of $10^{-9}$ Torr with the help of turbo molecular pump. Then pure oxygen gas $(5 \mathrm{~N})$ at a pressure of 100 Torr was filled in the chamber. A laser beam was focused through a focusing lens of focal length $50 \mathrm{~cm}$ and was incident at angle of $90^{\circ}$ with respect to target surface. The specimens were scanned with the help of DC motor. The area of $40 \mathrm{~mm} \times 2 \mathrm{~mm}$ was exposed to laser pulses by a scanning speed of $0.55 \mathrm{~mm} / \mathrm{s}$. Four targets were exposed to various pulses of 1200, 1800, 2400 and 3000 at a constant laser fluence of $3.4 \mathrm{~J} / \mathrm{cm}^{2}$ under oxygen environments.

Scanning Electron Microscope (SEM) (JEOL-JSM-6480) was used for investigating the surface morphology of unirradiated and laser irradiated specimens. The structural analysis of the pre- and post-irradiated specimens was performed by using X-ray diffractometer (X'Pert PRO MPD). The computer controlled 50 KN Universal Tensile testing Machine (UTM) (AG-1 Shimadzu) was used for the tensile testing of both unirradiated and laser irradiated brass. With the gauge length of $30 \mathrm{~mm}$ each sample was deformed with cross head speed of $1 \mathrm{~mm} / \mathrm{min}$. 
Microhardness measurements were carried out by pyramid-shaped diamond indenter (Zwick/Roell ZHU-5030) for a $50 \mathrm{~g}$ load applied for $15 \mathrm{sec}$ and data reported here represents the average of at least four measurements.

\section{Results and Discussion}

\subsection{Surface Morphology}

Figure 1(a) shows the SEM micrograph of an unexposed surface of brass. SEM micrographs of Figures 1(b)-(e) reveal the modified surface of brass after irradiation with a fluence of $3.4 \mathrm{~J} / \mathrm{cm}^{2}$ in an oxygen environment for various number of overlapping laser pulses of (b) 1200 (c) 1800, (d) 2400 and (e) 3000. Figure 1(b) shows that various features with non-uniform shape and density distribution are observed after exposing the surface with the laser pulse of 1200 . They include 1) micro-sized cavities surrounded by uplifted edges, 2) bumps, 3) cones and 4) wave-like ridges. When the number of pulses is increased to 1800 , a decline in the number density of cavities, bumps and wave-like ridges is observed (Figure 1(c)) whereas, the size of cavities increases significantly. An appearance of multiple ablative layers and ripples at the edges of cavities is also distinct and can be seen in the inset of Figure 1(c). The further increase in laser pulse up to 2400, the change in the surface morphology is observed in Figure 1(d). The appearance of wave-like ridges becomes more pronounced, whereas density of the bumps and cavities reduces significantly (Figure 1(d)). For the maximum number of pulse i.e. 3000 , the density of the ridges and bumps decreases whereas the density of cavities significantly increases as shown in Figure 1(e). The cracks are also generated for this number of pulses. The cavity-formation as seen in Figures 1(b)-(e) is due to mass removal after produced by the laser induced heating, thermal desorption, melting and explosive boiling of the irradiated surface [10] [11]. When the laser beam intensity exceeds a certain value than the ablation threshold, the target material begins to evaporate, resulting in to the formation of plasma. When the pressure of plasma is higher than the surrounding pressure, the liquefied material is expelled explosively from the irradiated surface due to the violent recoil pressure and becomes a possible cause for the formation of cavities, bumps and wave-like ridges [12].

Cumulative laser pulse irradiation and surface vaporization resistant impurities are responsible for the cone formation. The direction of these cones is forward peaked towards the incident laser radiation [13]. The interaction of laser pulses causes hydrodynamic instabilities at liquid-solid interface and also plays a significant role for the formation of wave-like ridges and cavities as seen in Figures 1(b)-(e) [14]. During laser matter interaction, surface plasmons are formed. The excitation of these plasmons induce an enhancement of local fields in the surface layer and causes the formation of ripples in surrounding of the cavities [15].

A decrease in number density of cavities is observed in Figure 1(d) with increasing number of laser pulses up to the range of 2400. Decrease in number of cavities can be due to the reason that shock liquidized material completely or partially refills the cavities produced by initial melting [16].

Features with non-uniform shape and size distribution are generated at the laser irradiated brass due to surface defects, inclusions, small pits, contaminants, oxides, laser induce thermal stresses and other heterogeneities. The growth of laser induced defects depends on higher sensitivity of laser absorption at that particular sites of material and therefore, causes the non-uniform laser energy absorption inside the material [17].

The cracks in Figure 1(e) are due to thermal stress cracking induced due to multiple pulse-irradiation , which possibly gives rise to breakage of atomic bonds at the irradiated surface [18]. The molten material is ejected from the irradiated surface and then cools on a relatively colder target area. As a result, the surface and subsurface layers exhibit laser induced temperature gradients and thermal stresses [16].

\subsection{XRD Analysis}

XRD technique is employed to attain phase identification, the variation in crystallinity, dislocation densities and residual stresses in the target material. Figure 2 shows the XRD patterns of unirradiated and laser irradiated brass targets. Figure 3 shows the variation in crystallite size with increasing number of laser pulses. Figure 4 shows a plot of variation of dislocation line density as a function of laser pulses. Untreated sample shows the presence of (320), (111), (332), (600), (210) and (102) planes reflection at angles of $41.7^{\circ}, 42.6^{\circ}, 48.59^{\circ}, 62.46^{\circ}$, $71.62^{\circ}$, and $78.03^{\circ}[19]$ respectively. It is also observed that d-spacing, peak intensity and the values of full width at half maximum (FWHM) changes after laser irradiation whereas no change in the phase is found. The absorption of non-uniform conduction of the energy by atoms, recrystallization, non-uniform thermal stresses, 

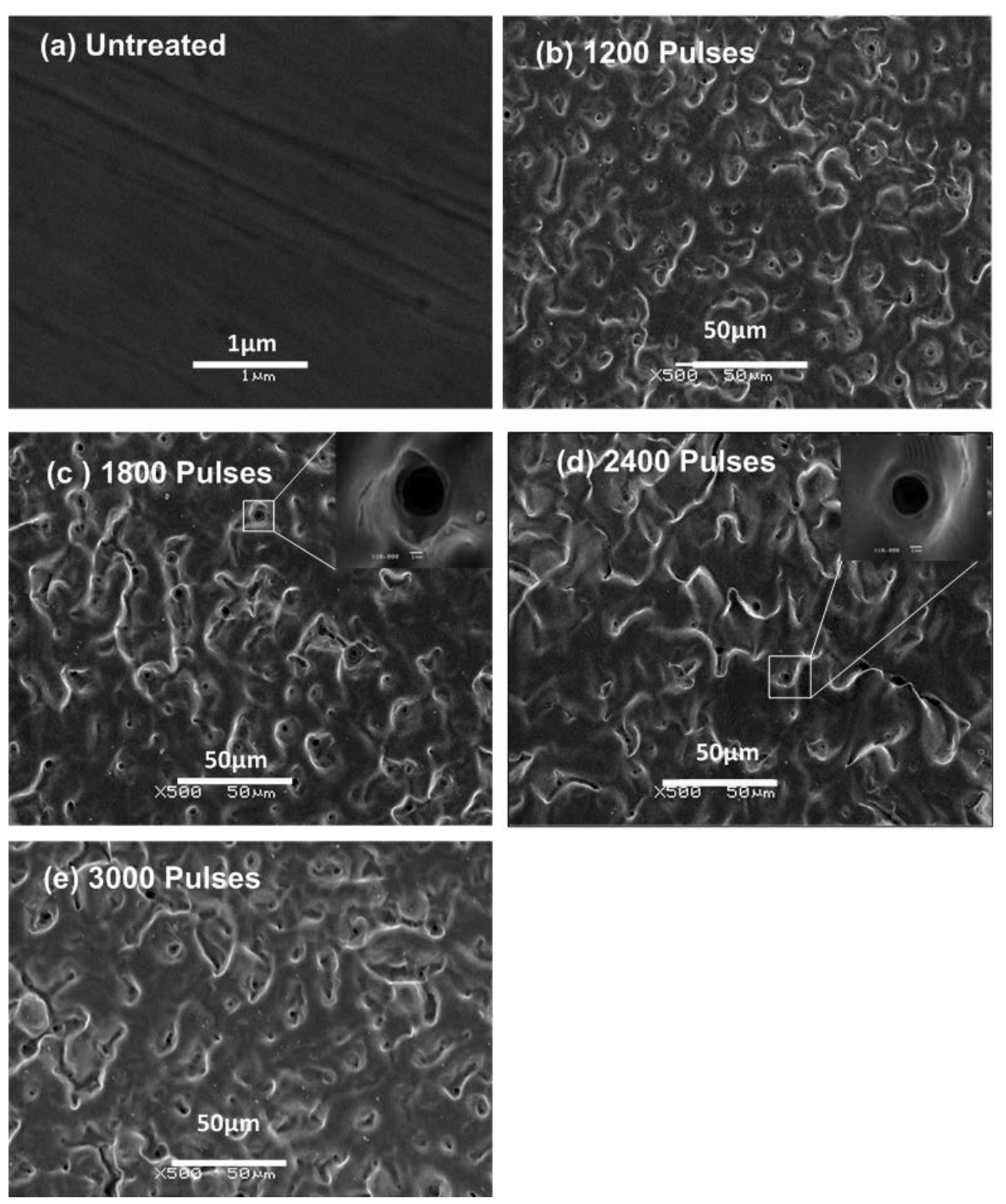

Figure 1. SEM images revealing the surface morphology of (a) unirradiated and Excimer laser irradiated brass for various number of pulses of (b) 1200, (c) 1800, (d) 2400 and (e) 3000.

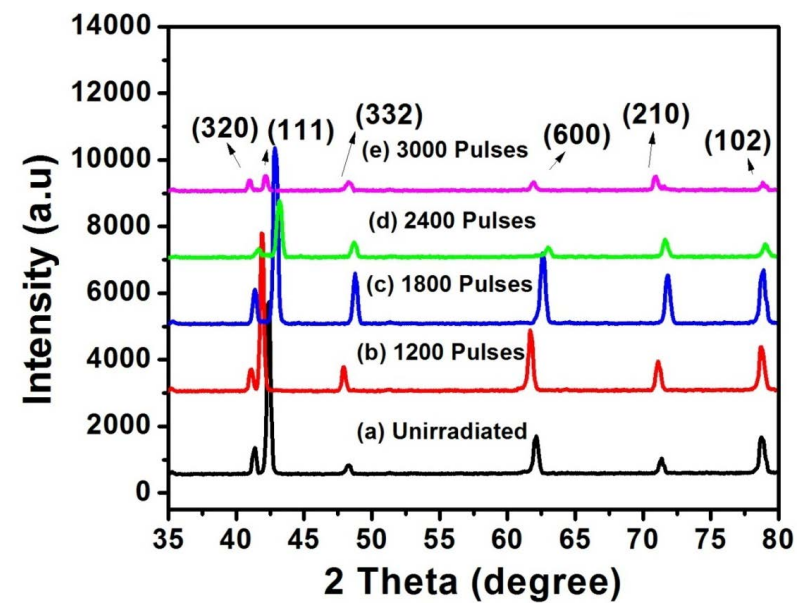

Figure 2. XRD patterns of (a) unirradiated and excimer laser irradiated brass for various number of pulses of (b) 1200, (c) 1800, (d) 2400 and (e) 3000. 


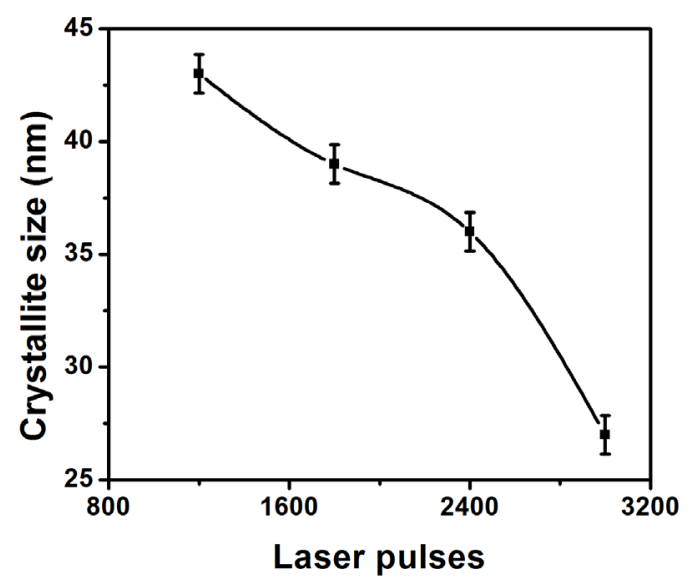

Figure 3. The variation of crystallite size of Excimer laser irradiated brass for various numbers of pulses of 1200, 1800, 2400 and 3000.

and scattering effect may cause variations in the peak intensity after the laser beam interaction with the brass target [9].

The crystallite size is evaluated for the reflection plane of brass (111) by using Sherrer's formula [16].

$$
\text { Crystallite } \operatorname{Size}(D)=\frac{0.9 \lambda}{\text { FWHM } \cos \theta}
$$

where $D$ is crystallite size, $\lambda$ is the wavelength of X-rays (1.542 $\AA$ ), FWHM is full width at half maximum, and $\theta$ is the angle of diffraction. FWHM and $\theta$ are measured in radian.

The residual strain variations and the dislocation line density are evaluated by using following relation [16].

$$
\begin{aligned}
\text { Dislocation Density } & =1 /(\text { Crystallite Size })^{2} \\
\operatorname{Strain}(\varepsilon) & =\frac{d-d_{0}}{d_{0}}
\end{aligned}
$$

where $d$ is the observed and $d_{0}$ is the standard plane spacing and $\varepsilon$ is the induced strain.

The laser induced stresses " $\sigma$ ” are calculated by using the relation given below [16].

$$
\operatorname{Stress}(\sigma)=\varepsilon E
$$

where $E$ is the young's modulus, and for brass its value is $102 \mathrm{GPa}$ [9].

The variation in the peak intensities and d-spacing for various laser pulses under oxygen background is demonstrated in Figure 2. The difference in the peak intensity is related to the variation in the crystallite size and laser induced strain on the target surface after laser irradiation. The change in $d$-spacing causes variation in residual stresses and lattice distortion. The differences in inter atomic distances, thermal expansion coefficients, heating and cooling conditions between the surface layers and interstitial diffusion can be a possible cause to produce lattice distortions in lattice planes [20].

Peak intensity for the plane of brass (111) (Figure 2(a)) increases with increasing number of laser pulses up to 1800. This increase in peak intensity is ascribed to the crystal growth and atomic diffusion of host material across the grain boundaries after laser ablation [21]. Further increase in number of pulses from 2400 to 3000, the reduction in peak intensity of plane is observed. The main reason for this decrease is recrystallization phenomenon is due to laser induced melting and resolidification. After laser irradiation large sized grains disintegrate into smaller sized which result an attenuation in peak intensity of (111) plane as shown in Figure 2(a). The crystallite size deceases with increase in number of pulses up to a maximum value of 3000 and is shown in Figure 3. Laser energy deposition to the target surface generates lattice strains by interstitial diffusion of host atoms. As a result large numbers of laser induced defect are produced which cause to reduce the crystallite size. Therefore these induced defects act as a barrier in dislocation motion [22]. Figure 4 depicts that an increasing trend in dislocation line density with increasing number of laser pulses, and it represents the increase in the concentration of the 


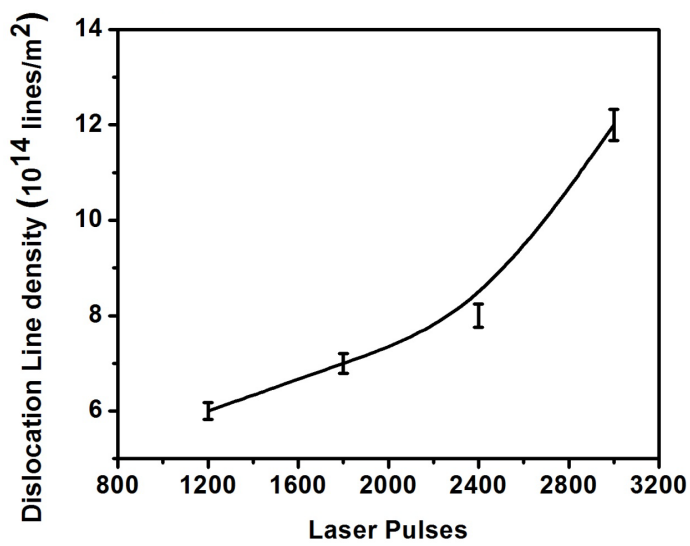

Figure 4. The variation in dislocation density of Excimer laser irradiated brass for various numbers of pulses of 1200, 1800, 2400 and 3000.

imperfections in lattice sites. The increase in the dislocation line density in the crystal lattice is due to enhanced quantification of work hardening [23]. The energy absorbed during laser material interaction is utilized not only to increase the mobility of existing dislocation defects but also to generate a great number of new dislocation defects by vibration or distortion [23]. The variation in laser induced stresses corresponding to various number of overlapping laser pulses is displayed in Figure 5. When high energy laser beam is incident on the solid target, lattice distortion and shock waves are generated. Consequently compressive and tensile stresses are produced in the irradiated brass. The high rates of heating and cooling also generate tremendous temperature gradients. This is associated with the local heating, and the plasma-dynamic flows. This is main reason for the production of residual stresses in the surface layer. The compressive stresses decrease and tensile stresses increase with increasing number of pulses [24]. These structural changes are well correlated with surface morphological modifications. SEM images of Figure 1 illustrate the formation of several kinds of features as cone, cavities and cracks. The cone formation is caused by the compressive stresses, whereas, cavities and crack formation is attributable to tensile stresses. The dislocation line density increases with increasing number of pulses up to 3000 (Figure 4) and consequently induces a high level of residual stresses in the irradiated target as observed in Figure 5 [25].

\subsection{Tensile Testing}

Figure 6 depicts stress-strain curves of 1) unirradiated and laser irradiated brass with a fluence of $3.4 \mathrm{~J} / \mathrm{cm}^{2}$ in an oxygen environment for various number of overlapping laser pulses of 2) 1200, 3) 1800, 4) 2400 and 5) 3000. The variations in the Yield Stress (YS) and the Ultimate Tensile Stress (UTS) with increasing number of laser pulses are shown in Figure 7 and Figure 8, respectively. Both the YS as well as UTS exhibit monotonic increase with increasing number of pulses. The laser induced defects, stresses and strain fields are possible reason for increasing behavior of YS and UTS with increasing number of pulses. These variations in the value of mechanical properties (YS and UTS) of brass are possibly attributed to the alterations in microstructure and dislocation density which is produced by laser beam-material interaction. As strengthening mechanism is directly proportional to the number of laser induced defects [26]. The impact of high energy photons of laser radiation creates initial displacement damage in the form of primary knock out atoms (PKA) which displace nearby atoms from their equilibrium position. The total numbers of displaced atoms in a cascade depends upon number of pulses, absorbed radiation energy, temperature and nature of target materials [27]. The generation of vacancies and interstitials, and/or their clusters, act as barriers to the glide dislocations. As the incident number of laser pulses increases, the defect density also increases. The increased in number of defects acts as strong pinning points for movement of dislocation plane. Hence dislocation movement reduces which causes improvement of the mechanical properties. For that reason greater mechanical stress will be necessary to start the plastic deformation [28].

\subsection{Microhardness}

Figure 9 shows the variation in the microhardness with increasing number of laser pulses. This graph also 


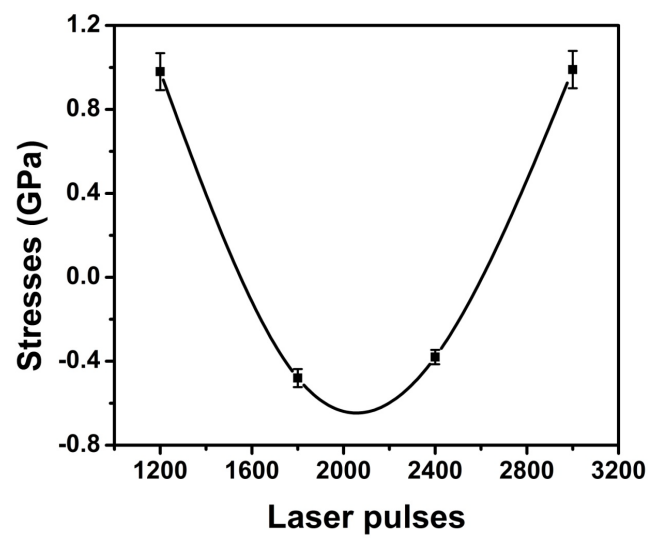

Figure 5. The variation of residual stress of Excimer laser irradiated brass for various numbers of pulses of 1200 , 1800, 2400 and 3000.

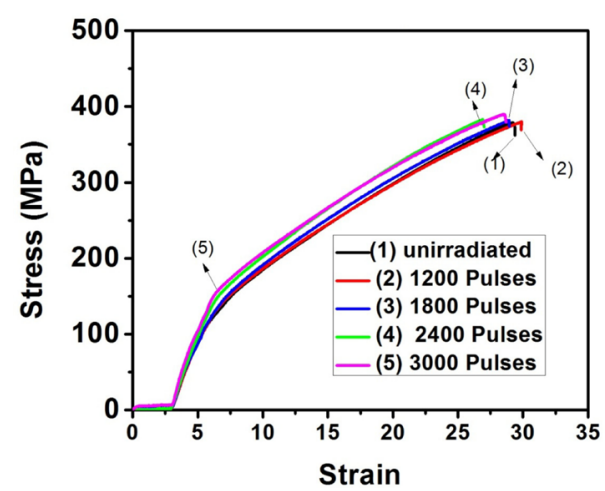

Figure 6. The comparison of stress-strain curves measured by tensile testing machine of unirradiated and Excimer laser irradiated brass for various numbers of pulses of 1200, 1800, 2400 and 3000.

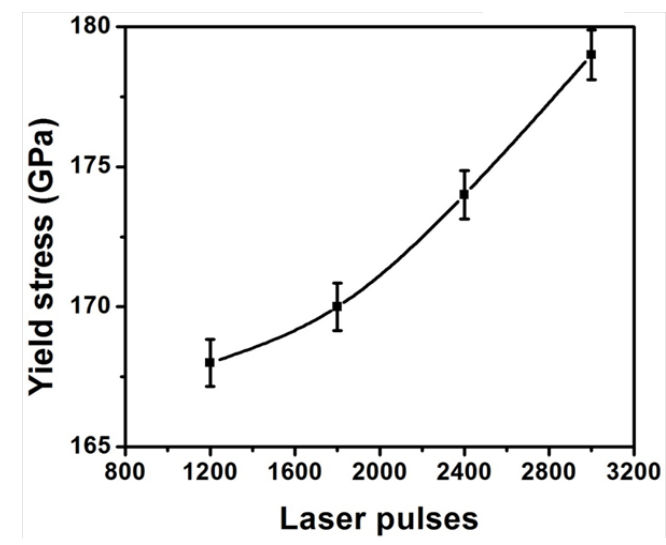

Figure 7. The variation of Yield Stress (YS) of excimer laser irradiated brass for various numbers of pulses of 1200, 1800, 2400 and 3000.

shows a monotonic increases in the microhardness of laser irradiated brass with increasing number of pulses. The changes in micro hardness are caused by the lattice disorder, associated with variation in crystal structure and laser induced thermal tensile stresses produced in the material. The alteration in microhardness can also be 


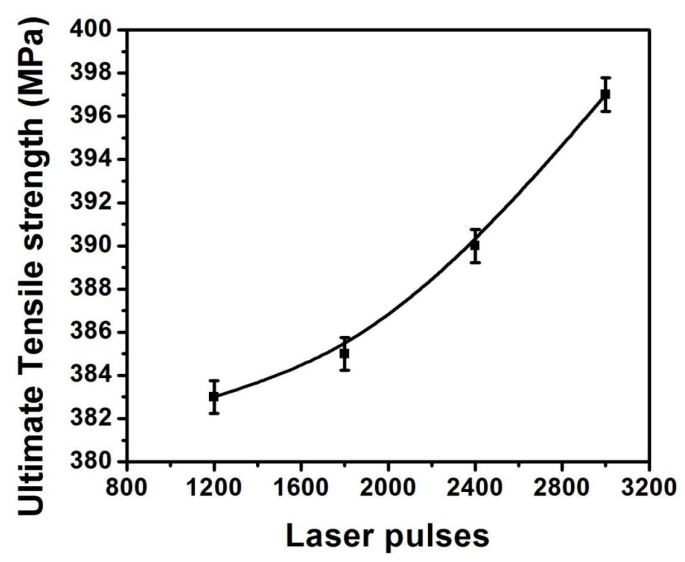

Figure 8. The variation of Ultimate Tensile Strength (UTS) of excimer laser irradiated brass for various numbers of pulses of 1200, 1800, 2400 and 3000.

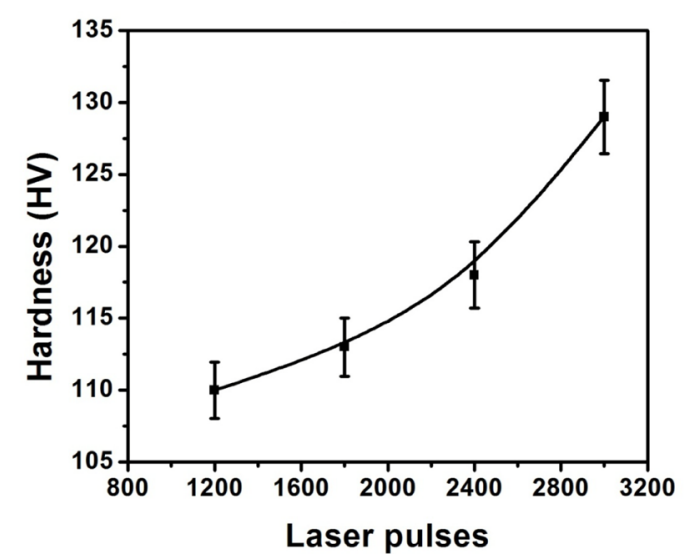

Figure 9. The variation in the microhardness of Excimer laser irradiated brass for various numbers of pulses of 1200, 1800, 2400 and 3000.

correlated with the change in crystalline size and dislocation density as has been calculated by XRD analysis and is shown in Figure 3 and Figure 4 respectively. By decreasing the grain size and increasing the dislocation density the microhardness increases [29].

\section{Conclusions}

The effect of nanosecond laser pulses on surface, structural and mechanical properties of brass has been investigated. After laser treatment laser-induced micro-sized cavities, bumps, cones and wave-like ridges with nonuniform shape and density distribution are formed. With increasing number of laser pulses waves-like ridges become less pronounced and more diffusive whereas cracks become dominant. The initial decreasing and then increasing trend in size and density of cavities with increasing number of laser pulses has been observed. The conversion of compressive residual stresses into tensile stresses with increasing number of pulses has also been observed. It is also revealed that the thermal stresses induced by laser irradiation were not enough to introduce new phases in target. The increasing trend in mechanical properties (YS, UTS and hardness) with increasing number of pulses is found. The changes in mechanical properties of irradiated brass specimen are well correlated with surface and crystallographical modifications.

\section{Acknowledgements}

The authors are grateful and acknowledge the help of Dr. Riaz (Director of CASP), Scanning Electron Micro- 
scope group and Mr. Sajjad (In charge of tensile testing lab) at Center for Advanced Studies in Physics, Govt. College University, Lahore, Pakistan.

\section{References}

[1] Jain, I.P. and Agarwal, G. (2011) Ion Beam Induced Surface and Interface Engineering. Surface Science Reports, 66, 77-172. http://dx.doi.org/10.1016/j.surfrep.2010.11.001

[2] Kim, H.S., Kim, W.Y. and Song, K.H. (2012) Effect of Post-Heat-Treatment in ECAP Processed Cu-40\%Zn Brass. Alloys and Compounds, 536S, S200-S203. http://dx.doi.org/10.1016/j.jallcom.2011.11.079

[3] Liu, X., Du, D. and Mourou, G. (1997) Laser Ablation and Micromachining with Ultrashort Laser Pulses. Journal of Quantum Electronics, 33, 1706-1716. http://dx.doi.org/10.1109/3.631270

[4] Bashir, S., Rafique, M.S. and Haq, F. (2007) Laser Ablation of Ion Irradiated CR-39. Laser and Particle Beams, 25, 181-191. http://dx.doi.org/10.1017/S0263034607070231

[5] Bashir, S., Vaheed, H. and Mahmood, K. (2013) Nanosecond Pulsed Laser Ablation of Brass in a Dry and Liquid-Confined Environment. Applied Physics, A110, 389-395. http://dx.doi.org/10.1007/s00339-012-7175-0

[6] Lin, Z.Z.P. and Ren, L. (2014) The Mechanical Properties and Microstructures of AZ91D Magnesium Alloy Processed by Selective Laser Cladding with Al Powder. Optics and Laser Technology, 60, 61-68. http://dx.doi.org/10.1016/j.optlastec.2013.12.024

[7] Kazakevich, P.V., Simakin, A.V., Shafeev, G.A., Monteverde, F. and Wautelet, M. (2007) Phase Diagrams of Laser-Processed Nanoparticles of Brass. Applied Surface Science, 253, 7724-7728. http://dx.doi.org/10.1016/j.apsusc.2007.02.176

[8] Tam, K.F., Cheng, F.T. and Man, H.C. (2002) Laser Surfacing of Brass with Ni-Cr-Al-Mo-Fe Using Various Laser Processing Parameters. Materials Science and Engineering A, 325, 365-374.

http://dx.doi.org/10.1016/S0921-5093(01)01484-8

[9] Ahmad, S., Bashir,S., Ali, N., Kalsoom, U., Yousaf, D., Haq, F., Naeem, A., Ahmad, R. and Rahman M.K. (2014) Effect of Ion Irradiation on the Surface, Structural and Mechanical Properties of Brass. Nuclear Instruments and Methods in Physics Research B, 325, 5-10. http://dx.doi.org/10.1016/j.nimb.2014.01.023

[10] Perez, D. and Lewis, L.J. (2002) Ablation of Solids under Femtosecond Laser Pulses. Physical Review Letters, 89, 255504-255508. http://dx.doi.org/10.1103/PhysRevLett.89.255504

[11] Chrisey, D.B. and Hubler, G.K. (1994) Pulsed Laser Deposition of Thin Films. John Wiley \& Sons Inc., Hoboken.

[12] Korner, C., Mayerhofer, R., Hartmann, M. and Bergmann, H.W. (1996) Physical and Material Aspects in Using Visible Laser Pulses of Nanosecond Duration for Ablation. Applied Physics A, 63, 123-131. http://dx.doi.org/10.1007/BF01567639

[13] Sanchez, F., Morenza, J.L., Aguiar, R., Delgado, J.C. and Verela, M. (1996) Structure Growth on Silicon Exposed to ArF Excimer Laser Irradiation. Applied Physics Letters, 69, 620-622. http://dx.doi.org/10.1063/1.117926

[14] Kang, H.W., Lee, H. and Welch, A.J. (2008) Laser Ablation in a Liquid-Confined Environment Using a Nanosecond Laser. Journal of Applied Physics, 103, Article ID: 083101. http://dx.doi.org/10.1063/1.2905314

[15] Bashir, S., Rafique, M.S. and Husinsky, W. (2012) Femtosecond Laser-Induced Subwavelength Ripples on Al, Si, CaF2 and CR-39. Nuclear Instruments and Methods in Physics Research Section B: Beam Interactions with Materials and Atoms, 275, 1-6. http://dx.doi.org/10.1016/j.nimb.2011.12.016

[16] Ali, N., Bashir, S., Kalsoom, U., Akram, M. and Mahmood, K. (2013) Effect of Dry and Wet Ambient Environment on the Pulsed Laser Ablation of Titanium. Applied Surface Science, 270, 49-57. http://dx.doi.org/10.1016/j.apsusc.2012.12.049

[17] Dauscher, A., Feregotto, V., Cordier, P. and Thorny, A. (1996) Laser Induced Periodic Surface Structures on Iron. Applied Surface Science, 96, 410-414. http://dx.doi.org/10.1016/0169-4332(95)00495-5

[18] Truong, S.L., Levi, G., Verduraz, F.B., Petrovskaya, A.V., Simakin, A.V. and Shafeev, G.A. (2007) Generation of Nanospikes via Laser Ablation of Metals in Liquid Environment and Their Activity in Surface-Enhanced Raman Scattering of Organic Molecules. Applied Surface Science, 254, 1236-1239. http://dx.doi.org/10.1016/j.apsusc.2007.07.157

[19] Wang, Z., Zhu, L., Li, W., Xu, H. and Liu, H. (2013) Superhydrophobic Surfaces on Brass with Controllable Water Adhesion. Surface \& Coatings Technology, 235, 290-296. http://dx.doi.org/10.1016/j.surfcoat.2013.07.054

[20] Choi, D., Shinavski, R.J., Steffier, W.S. and Spearing, S.M. (2005) Residual Stress in Thick Low-Pressure Chemical-Vapor Deposited Polycrystalline SiC Coatings on Si Substrates. Journal of Applied Physics, 97, Article ID: 074904. http://dx.doi.org/10.1063/1.1866495

[21] Latif, A., Rehman, M.K., Rafique, M.S. and Bhatti, K.A. (2011) Surface Morphologic and Structural Analysis of IR 
Irradiated Silver. Physica B: Condensed Matter, 406, 1713-1716. http://dx.doi.org/10.1016/j.physb.2011.02.013

[22] Wang, L., Ji, S. and Sun, J. (2006) Effect of Nitriding Time on the Nitrided Layer of AISI 304 Austenitic Stainless Steel. Surface \& Coatings Technology, 200, 5067-5070. http://dx.doi.org/10.1016/j.surfcoat.2005.05.036

[23] Rahman, M.K., Butt, M.Z., Samuel, A. and Siraj, K. (2010) Investigation of Laser Irradiation Effects on the Hardness of Al 5086 Alloy under Different Conditions. Vacuum, 85, 474-479. http://dx.doi.org/10.1016/j.vacuum.2010.08.025

[24] Shanmugan, S. and Mutharasu, D. (2012) An Effect of $\mathrm{N}^{+}$Ion Bombardment on the Properties of CdTe Thin Films. Radiation Physics and Chemistry, 81, 201-207. http://dx.doi.org/10.1016/j.radphyschem.2011.09.016

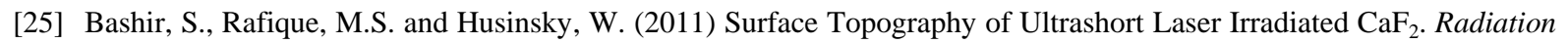
Effects and Defects in Solids: Incorporating Plasma Science and Plasma Technology, 166, 30-34. http://dx.doi.org/10.1080/10420150.2010.531726

[26] Song, B., Dong, S., Deng, S., Liao, H. and Coddet, C. (2014) Microstructure and Tensile Properties of Iron Parts Fabricated by Selective Laser Melting. Optics and Laser Technology, 56, 451-460. http://dx.doi.org/10.1016/j.optlastec.2013.09.017

[27] Jung, P. and Ullmaier, H. (1990) Effects of Light-Ion Irradiation on Mechanical Properties of Metals and Alloys. Journal of Nuclear Materials, 174, 253-263. http://dx.doi.org/10.1016/0022-3115(90)90239-J

[28] Ghauri, I.M. and Afzal, N. (2007) Effects of Neutron Irradiation on the Stress Relaxation Rate in Al-Cu-Mg Alloy. Journal of Physics D: Applied Physics, 40, 6044-6047. http://dx.doi.org/10.1088/0022-3727/40/19/041

[29] Wang, C., Zhou, H., Zhang, Z., Zhao, Y., Cong, D., Meng, C., Zhang, P. and Ren, L. (2013) Mechanical Property of a Low Carbon Steel with Biomimetic Units in Different Shapes. Optics \& Laser Technology, 47, 114-120. http://dx.doi.org/10.1016/j.optlastec.2012.07.037 
Scientific Research Publishing (SCIRP) is one of the largest Open Access journal publishers. It is currently publishing more than 200 open access, online, peer-reviewed journals covering a wide range of academic disciplines. SCIRP serves the worldwide academic communities and contributes to the progress and application of science with its publication.

Other selected journals from SCIRP are listed as below. Submit your manuscript to us via either submit@scirp.org or Online Submission Portal.
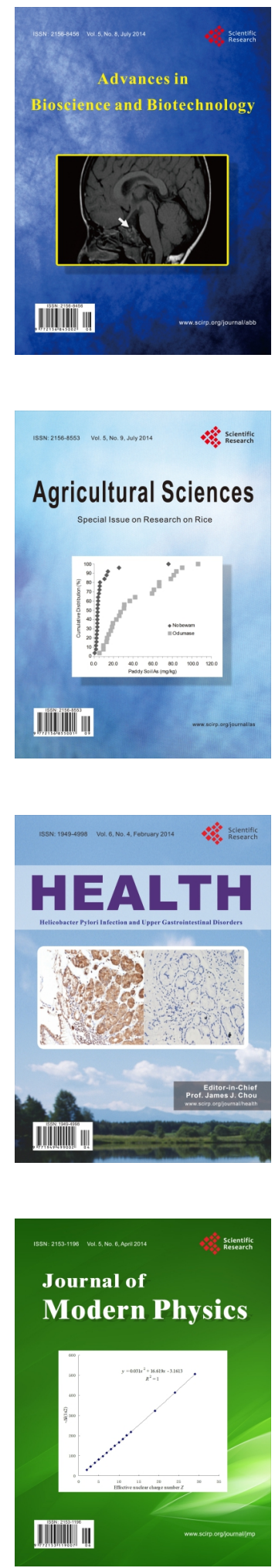
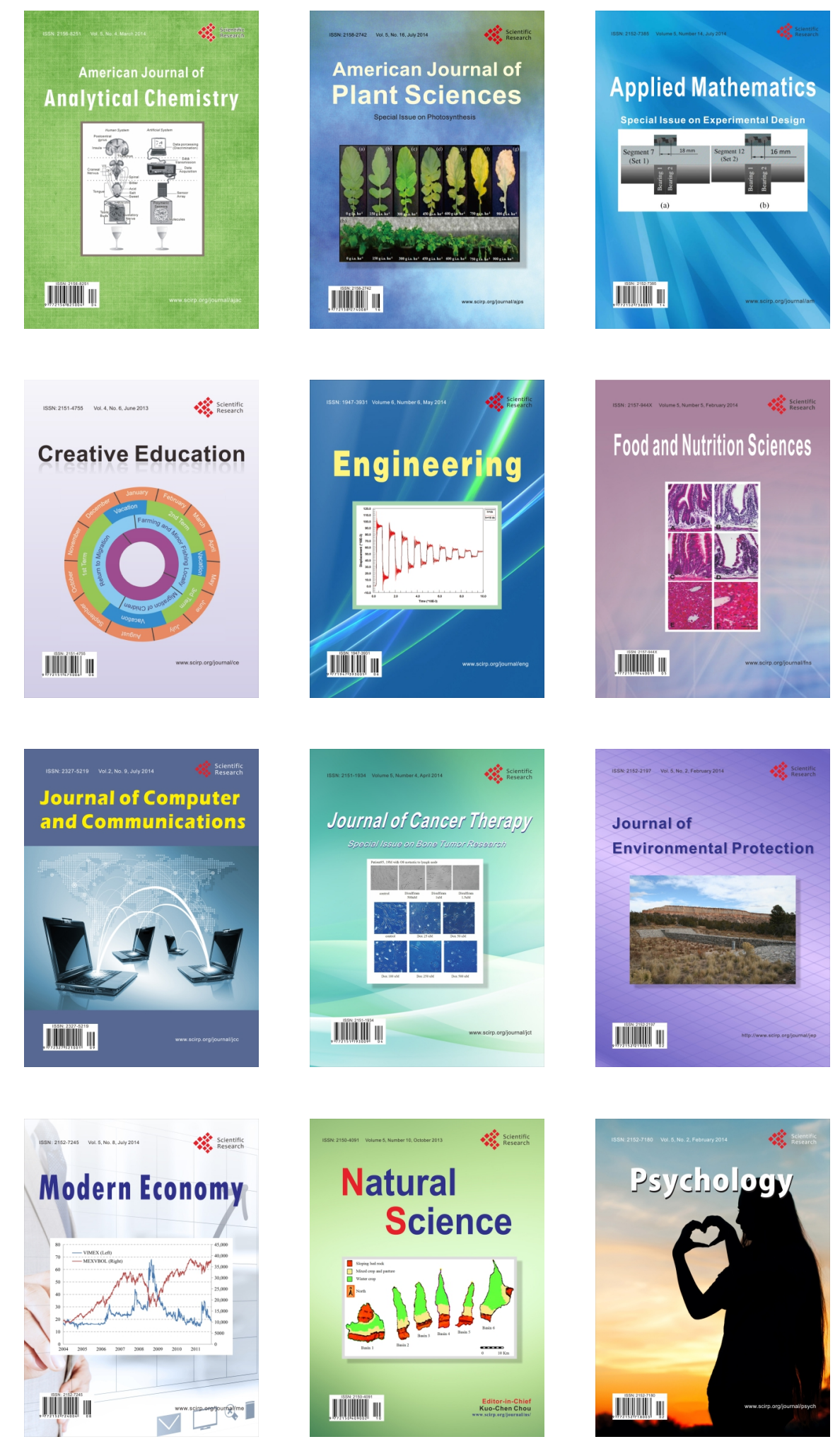